\title{
ELABORACIÓN DE UN SIMULADOR CON GEOGEBRA PARA LA ENSEÑANZA DE LA FÍSICA. EL CASO DE LA LEY DE COULOMB
}

\section{BUILDING OF A SIMULATOR WITH GEOGEBRA FOR TEACHING PHYSICS. THE CASE OF COULOMB LAW}

Ivonne C. Sánchez S. ${ }^{1}$

ORCID iD: $\underline{0000-0002-2485-1059}$

Irene V. Sánchez Noroño. ${ }^{2}$

ORCID iD: $\underline{0000-0001-9176-0125}$

\begin{abstract}
RESUMEN
Con la llegada del siglo XXI es más notorio el uso de tecnologías digitales en la enseñanza de la Matemática y de las Ciencias Naturales, uno de los tipos de recursos más usados son los simuladores computacionales. Las investigaciones con foco en las implicaciones del uso de estos recursos digitales en la enseñanza poco comentan sobre las potencialidades de la elaboración de simuladores para el aprendizaje y mucho menos de la matemática que emerge en esta actividad. Atendiendo a esta consideración, en este trabajo se describe la secuencia de las tareas de simulación resueltas con GeoGebra que permiten emular uno de los fenómenos electrostáticos de atracción y repulsión, vinculados a la Ley de Coulomb. La descripción incluye la especificación de los contenidos matemáticos emergentes en la actividad, así como una valoración subjetiva de las potencialidades del GeoGebra para el aprendizaje de los fenómenos físicos y los contenidos matemáticos emergentes de la experiencia.
\end{abstract}

Palabras clave: Elaboración de simuladores. GeoGebra. Matemática. Resolución de tareas.

\begin{abstract}
With the arrival of the 21st century, the use of digital technologies in the teaching of Mathematics and Natural Sciences is more noticeable. One of the most used types of resources is computer simulators. Research focused on the implications of the use of these digital resources in teaching little comment on the potential of the development of simulators for learning, much less the mathematics that emerges in this activity. Based on this consideration, this work describes the sequence of simulation tasks solved with GeoGebra that allow one to emulate one of the electrostatic phenomena of attraction and repulsion, linked to Coulomb's Law. The description includes the specification of the emerging mathematical content in the activity, as well as a subjective assessment of the potential of GeoGebra for learning physical phenomena and the emerging mathematical content of the experience.
\end{abstract}

Keywords: Elaboration of simulators. GeoGebra. Mathematics. Task solving.

\footnotetext{
${ }^{1}$ Magíster en Educación en Ciencias y Matematica por la Universidad Federal de Pará (UFPA). Coordinadora Administrativa de la Asociación Aprender en Red, Maracaibo, Zulia, Venezuela. Dirección para correspondencia: Calle 65B, No 95-29C, barrio Guaicaipuro, Maracaibo, Estado Zulia, Venezuela, Dirección postal: 4001. E-mail: ivonne.s.1812@gmail.com.

${ }^{2}$ Magíster Scientiarum en Matemática mención Docencia por la Universidad del Zulia (LUZ). Académica de la Universidad Arturo Part (UNAP), Iquique, Tarapacá, Chile. Av. Arturo Part 2120, DP 1100000. Coordinadora de Investigación de la Asociación Aprender en Red, Maracaibo, Zulia, Venezuela. Dirección para correspondencia: Calle 65B, No 95-29C, barrio Guaicaipuro, Maracaibo, Estado Zulia, Venezuela, Dirección postal: 4001. E-mail: irsanchez@unap.cl.
} 


\section{INTRODUCCIÓN}

La rápida evolución que han mostrado las tecnologías digitales en los últimos años deja ver la importancia de que éstas sean parte del proceso educativo en todos los niveles, en tanto que son consideradas como un medio potencial y necesario para mejorar la calidad educativa en las diferentes áreas de conocimiento (UNESCO, 2015). Particularmente, la influencia de las tecnologías digitales ha sido más notoria en las áreas de las Matemáticas y las Ciencias Naturales, debido a que han permitido la creación de recursos educativos digitales de diversos tipos (GÓMEZ, 2017), que proporcionan condiciones para que los aprendices identifiquen, examinen y comuniquen sus ideas matemáticas, físicas, químicas, entre otras. A su vez, algunos de éstos recursos han favorecido el estudio experimental de las ciencias, lo que posibilita la generación y validación de conjeturas matemáticas o científicas, conllevando a que los estudiantes tengan una comprensión adecuada de los contenidos abordados en las áreas mencionadas (GAMBOA, 2007; VILLAREAL, 2012).

Algunos recursos digitales que han sido elaborados con tecnologías digitales son los simuladores y videojuegos, los cuales han favorecido el aprendizaje tanto en matemáticas como en ciencias naturales, revelando así un gran potencial para promover la comprensión conceptual, la motivación y el desarrollo del discurso científico en torno a fenómenos que, en la realidad, sería imposible observar y manipular (BAEK, 2009; FEITOSA; LAVOR, 2020; HONEY; HILTON, 2011). Sin embargo, la mayoría de las investigaciones relacionadas con esta temática se enfocan en el uso de los simuladores como recursos didácticos que coadyuvan a la comprensión y estímulo hacia el estudio de las matemáticas y las ciencias naturales (BURBANO; PINTO; VALDIVIESO, 2015), dejando de lado la elaboración de estos recursos como una actividad desde la cual es posible que ocurra aprendizaje matemático, en un entorno de descubrimiento y reflexión.

Trabajos recientes han reportado las matemáticas y la física que subyace en la elaboración de simuladores computacionales (CASTILLO; PRIETO; SÁNCHEZ; GUTIÉRREZ, 2019; GUTIÉRREZ; PRIETO; ORTIZ, 2017; RUBIO, PRIETO; ORTIZ, 2016; SÁNCHEZ; PRIETO 2017), en los cuales se enfatiza la importancia de considerar los referentes teóricos de la matemática y de la física que permiten la elaboración del simulador en la tecnología seleccionada (CERVANTES; RUBIO; PRIETO, 2015). Un medio tecnológico que se ha mostrado potente para promover la apropiación de estos referentes teóricos es el GeoGebra, un software de matemática dinámica y de código abierto que integra múltiples representaciones de los objetos matemáticos en un mismo entorno, lo que favorece la 
exploración, construcción y validación de modelos matemáticos (HOHENWARTER; PREINER, 2007).

En este sentido, la elaboración de simuladores con GeoGebra es una actividad que es llevada a cabo por etapas, de acuerdo a un conjunto de tareas de simulación que se vinculan a la representación de algún aspecto del fenómeno que se intenta simular en el software (RUBIO; PRIETO; ORTIZ, 2016). Durante la resolución de estas tareas surgen diversos contenidos matemáticos que les dan respuesta, dando pie a un nuevo tipo de tarea más matemática (por así decirlo), denominada tarea de construcción (SÁNCHEZ; PRIETO, 2017). Las tareas de construcción se orientan al estudio y representación de modelos matemáticos que emulan las partes del fenómeno que se desea representar en el GeoGebra. No obstante, la elaboración de este tipo de simulador es una actividad que se ha venido desarrollando de manera progresiva, por lo que aún en la actualidad existen interrogantes por responder, con respecto a las condiciones de esa actividad, el alcance y las limitaciones del software para elaborar tales simuladores.

Por lo antes expuesto, y con el propósito de realizar contribuciones a la comprensión de la actividad de elaborar un simulador con GeoGebra, en este trabajo describimos las ideas matemáticas que emergieron en la resolución de un conjunto de tareas de simulación que fueron atendidas para elaborar un simulador con GeoGebra sobre la Ley de Coulomb. Con ello buscamos atender un doble propósito: por un lado, reportar los contenidos matemáticos empleados en la construcción del simulador que emula los fenómenos electrostáticos, mientras que por otro lado, destacar las potencialidades del GeoGebra para construir simuladores.

\section{CONTEXTO DE LA EXPERIENCIA}

La elaboración del simulador que se describe surgió como un requerimiento de la Práctica Profesional III $^{3}$ que la primera autora realizó en el segundo periodo del 2015 (de octubre 2015 a marzo 2016). El requerimiento consistió en realizar una práctica de laboratorio de Física, mediada por tecnologías digitales, en una institución educativa oficial de Venezuela. Como parte del proceso que implicaba atender al requerimiento descrito, la primera autora participó de una experiencia formativa orientada hacia la elaboración de simuladores con el

\footnotetext{
${ }^{3}$ La práctica profesional III es una disciplina en la cual se da la oportunidad a los futuros profesores para aplicar en alguna institución educativa, aquellos procedimientos didácticos pedagógicos que contextualizan los saberes aprendidos durante su formación.
} 
software GeoGebra para la enseñanza de las matemáticas y de las ciencias naturales. Esa experiencia formativa, ofrecida por la Asociación Aprender en Red y el Instituto GeoGebra de Maracaibo (para el año), fue realizada en el mes de octubre del año 2015 en una institución educativa oficial de la ciudad de Maracaibo (Zulia, Venezuela), bajo la orientación de una formadora con experticia en la temática (Ver Figura 1).

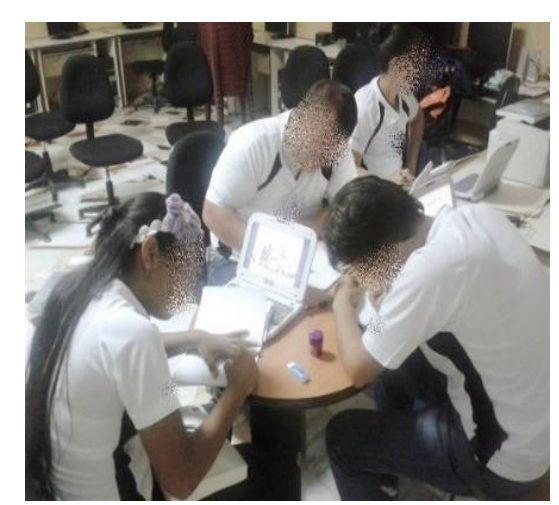

Figura 1 - Jornadas de Elaboración de Simuladores con GeoGebra. Fuente: Fotografía tomada por las autoras

Así, el simulador del cual se hace mención en este trabajo fue el producto que la primera autora obtuvo para aprobar satisfactoriamente la experiencia formativa comentada. Dicho simulador se elaboró con el propósito de abordar un contenido físico específico que estuviese contemplado en el plan de estudios de la Educación Media (12-17 años) del Subsistema de Educación Básica de Venezuela, en el que la primera autora se desempeñaba como docente para el momento. El contenido seleccionado para elaborar el simulador con GeoGebra fue la Ley de Coulomb.

\section{DESCRIPCIÓN DE LA ESCENA DEL SIMULADOR}

El simulador elaborado recrea una escena en la cual se visualiza cómo se carga eléctricamente un peine por frotamiento, esto es, frotar el peine con un pañuelo de lana por unos segundos, una vez cargado, se acerca a un grupo de papelillos que consideramos poseen carga neta cero, es decir, que poseen la misma cantidad de electrones y protones. Cuando el peine cargado negativamente se acerca a los trozos de papel, los electrones de los átomos que los componen tienden a alejarse temporalmente de la zona cargada negativamente del peine. Simultáneamente, la zona de los papelillos más próxima al peine queda cargada positivamente, lo que permite que algunos papelillos se adhieran al peine debido a la fuerza eléctrica de atracción que éste ejerce sobre los papelillos. Lo anterior se conoce en Física como el fenómeno 
de polarización eléctrica. Luego de transcurrido un tiempo, los electrones del peine son transferidos a los trozos de papel debido a la electrización por contacto. Como ambos objetos (peine y papelillos) tienen cargas del mismo signo, se manifiesta entre ellos la fuerza eléctrica de repulsión.

\section{CONSIDERACIONES DE LA ELABORACIÓN DE LA SIMULACIÓN}

Para la elaboración de la simulación se tomaron en cuenta algunas consideraciones de carácter teórico, curricular y didáctico, que permitieran crear un recurso de la Ley de Coulomb y que sirvieran de base para las decisiones y acciones en la elaboración. En lo siguiente, se describen cada una de estas consideraciones:

4.1 Consideraciones teóricas: En lo teórico, se tomaron en cuenta algunos criterios que se consideraron necesarios para realizar el simulador, de manera que éste respondiese al fenómeno que expresa la Ley de Coulomb.

- El primer criterio aborda tres aspectos inherentes al fenómeno a simular, a saber: (i) la Fuerza Eléctrica, ésta es, la fuerza que puede ejercer un cuerpo que está cargado sobre otro, el cual puede estar cargado eléctricamente o no. En experimentos electrostáticos realizados con materiales concretos, se puede visualizar solo la fuerza de atracción. Lo anterior se debe a los procesos de electrización de los cuerpos; (ii) El enunciado de la "Ley de Coulomb", que expresa que "la magnitud de la fuerza eléctrica es proporcional al producto de las cargas e inversamente proporcional al cuadrado de la distancia entre las cargas". Esta ley determina la intensidad de la fuerza eléctrica que ejerce una carga sobre otra mediante la fórmula matemática $F_{e}=K \frac{\left|q_{1}\right|\left|q_{2}\right|}{r^{2}} N$; y (iii) La magnitud vectorial de la fuerza, en este caso, el vector unitario que indica el sentido de la fuerza eléctrica que ejerce una carga sobre otra (SERWAY, 1997). Cuando las cargas son de igual signo las fuerzas serán de repulsión, por lo que el sentido el vector unitario coincide con el vector de fuerza resultante que ejerce una carga sobre la otra. En cambio, si las cargas son de signos opuestos, las fuerzas serán de atracción, siendo el sentido del vector unitario opuesto al vector fuerza resultante de la interacción, lo que hemos denominado en el simulador como la representación matemática de la escena.

- El segundo criterio responde a los procesos físicos que interactúan en la escena simulada. Uno de ellos es la electrización, que consiste en dotarle a un cuerpo 
propiedades eléctricas, es decir, que el cuerpo adquiera cargas eléctricas. En la electrostática existen distintas maneras de electrizar un cuerpo. Una es la electrización por frotamiento, la cual se produce cuando se frotan materiales con distinta capacidad para retener electrones. Cuando este tipo de electrización ocurre, cada uno de los cuerpos que se rozan quedan cargados con cargas de distinto signo. Otra es la electrización por contacto, que se origina cuando se pone en contacto un cuerpo con otro previamente electrizado. En este caso ambos cuerpos quedan cargados con cargas del mismo signo. Por último está la electrización por inducción, que no requiere de contacto directo entre los materiales. Se produce cuando se acerca un cuerpo cargado eléctricamente a otro que se encuentra neutro. En esa situación, es generada una interacción eléctrica entre las cargas del objeto electrizado y las del cuerpo neutro, dando como resultado que la distribución de cargas se altere, pues el cuerpo electrizado induce una carga con signo contrario en la parte más próxima del cuerpo neutro y por lo tanto lo atrae. Este fenómeno en física se conoce como polarización eléctrica.

- El tercero se relaciona con las propiedades de las cargas eléctricas: (i) La carga se conserva, esto es, no se crea ni se destruye, sino que simplemente se transfiere de un cuerpo a otro; (ii) La carga esta cuantizada, o sea, que la mínima cantidad de carga eléctrica equivale a la carga de un electrón y la carga eléctrica en cualquier cuerpo es submúltiplo entero de la carga del electrón; (iii) La naturaleza de la carga, que puede ser positiva o negativa.

4.2 Consideraciones curriculares: En cuanto a lo curricular, el contenido de la Ley de Coulomb está presente en los programas vigentes de Física de nivel medio en Venezuela, específicamente, en el quinto año (16-17 años). En los planes de estudio se sugiere que los estudiantes deben ser capaces de caracterizar la Ley de Coulomb y vivir experiencias donde relacionen la realidad con los fenómenos físicos que observan para movilizar sus procesos y operaciones mentales. Adicional a ello, en los libros didácticos más actuales que utilizan los profesores de matemáticas y física de Venezuela se sugiere que deben realizarse experimentos relacionados con la electrostática, a fin de comprender las interacciones de las fuerzas eléctricas y la Ley de Coulomb. En base a los planteamientos del programa y las sugerencias de los libros didácticos, se decidió realizar un simulador en el cual se pueda promover en los estudiantes la comprensión de las interacciones entre dos cargas y el enunciado de la Ley de Coulomb. 
4.3 Consideraciones didácticas: Con respecto a lo didáctico, se buscó crear un escenario en el cual fuese posible, por un lado, que el estudiante se visualizase en el experimento el frotamiento entre el peine con el pañuelo de lana y luego aproximarlo a los papelillos y, por otro lado, mostrar el concepto físico de la Ley de Coulomb que cuantifica las fuerzas presentes en el experimento junto al diagrama de fuerzas vectoriales. Además, se buscó hacer posible que el estudiante pudiera interactuar con el simulador, dado que el valor de la cargar se puede variar -en un determinado rango-, modificándose el valor de la fuerza, permitiendo que los estudiantes puedan hacer conjeturas y comprobar las relaciones abordadas.

\section{DESCRIPCIÓN DE LA RESOLUCIÓN DE LAS TAREAS DE SIMULACIÓN}

En este apartado se describen la manera como fueron abordadas las tareas de simulación que tuvieron que ser resueltas para elaborar el simulador de la Ley de Coulomb con GeoGebra. Esas tareas responden a dos propósitos. El primero se relaciona con la elaboración de elementos dinámicos que dan sentido a la escena que se representa, como el frotamiento del peine con el pañuelo. El segundo propósito se refiere a los elementos decorativos de la escena y el panel de control del simulador, que permite manipular las variables del fenómeno. En total se realizaron seis tareas de simulación, siendo las tareas 2, 3 y 4 correspondientes al primer propósito, mientras que las tareas 1,5 y 6 vinculadas al segundo.

Antes de continuar, es preciso destacar que en la resolución de las tareas de simulación emergió una diversidad de contenidos matemáticos que permitió representar los elementos tantos dinámicos como decorativos. Así, para elaborar el simulador se utilizó la aplicación gráfico, dadas las facilidades que ésta brinda para introducir y manipular objetos matemáticos. Es importante mencionar que todas las medidas de longitud mencionadas a continuación tienen como base la unidad de medida predeterminada por el software.

\section{1: Inserción de imágenes (Tarea No. 1)}

La resolución de esta tarea inicia la elaboración del simulador. Su propósito fue insertar dos imágenes en la vista gráfica del GeoGebra: la primera imagen es de un pañuelo de lana y la segunda es un grupo de papelillos. Ambas imágenes debían tener una posición absoluta en la pantalla, lo que equivale a que las imágenes debían permanecer fijas en la pantalla. El GeoGebra está programado para que sus imágenes tengan una forma rectangular al insertarlas en su interfaz, forma geométrica que posee dos puntos en sus esquinas inferiores de forma predeterminada -que pueden ser modificadas-, lo que permite al usuario posicionar las 
imágenes de acuerdo a su preferencia. En este sentido, se requería conocer la ubicación de los puntos para posteriormente fijar las imágenes, para lo cual se debía localizar los puntos a partir del plano cartesiano, que posteriormente se asociarían a las esquinas inferiores de cada imagen. El plano cartesiano sirvió como referencia en la vista gráfica del software.

En relación con la imagen del pañuelo de lana, ésta se posicionó en el primer cuadrante del plano cartesiano. Para ello, se trazaron tres rectas paralelas, dos al eje $y$ y una al eje $x$ utilizando la barra de entrada del programa, introduciendo las expresiones $x=4, x=6.5 \mathrm{y}$ $y=1$, obteniendo las rectas $e, d_{1}$ y $c$, respectivamente. Luego se interceptaron las rectas $c$ con $d_{1}$ y $c$ con $e$, con la ayuda de la herramienta Intercepción, de lo cual se obtuvieron los puntos $A=(4,1)$ y $B=(6.5,1)$. Una vez determinados los puntos se asoció la imagen (paño de lana) a éstos, específicamente, el punto $A$ con la esquina inferior izquierda y el punto $B$ con la esquina inferior derecha. De forma análoga se determinaron los puntos para la imagen de los papelillos, solo que ésta se posicionó en el tercer cuadrante y se consideró al Ejey como una recta.

Para lo anterior, se trazaron las rectas $x=-2,5$ y $y=-2,5$, llamadas respectivamente $i$ y $j$ que luego se interceptaron generando el punto $C=(-2.5,-2.5)$ y la recta $j$ con el Ejey, determinado el punto $D=(0,-2.5)$. Estos puntos se emplean para ubicar la imagen (papelillos) al tercer cuadrante, asignando a la esquina inferior izquierda $C$ y la derecha el punto $D$ (Ver Figura 2).

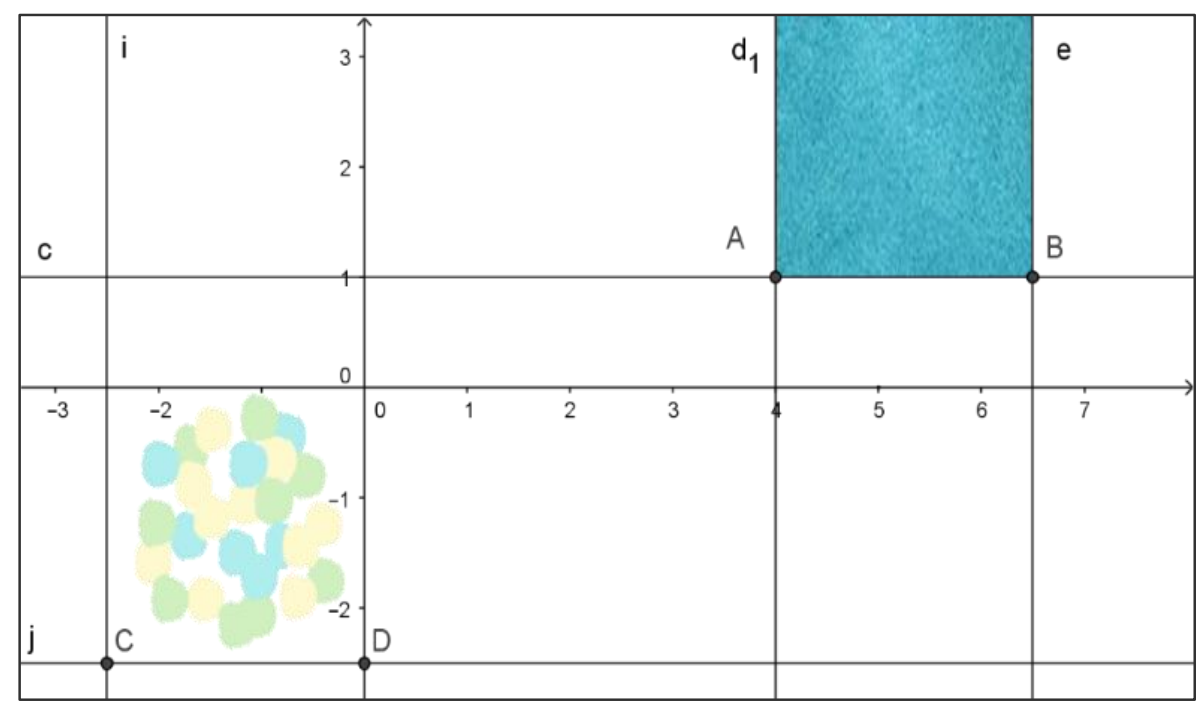

Figura 2 - localización en el plano de las imágenes (papelillos y paño de lana) Fuente: Elaborado por las autoras

\subsection{Movimiento para cargar el peine (Tarea No. 2)}

El propósito de esta tarea fue recrear el movimiento que permite cargar el peine por frotamiento. Para lograr esto, fue necesario determinar un punto que tuviera un movimiento 
vertical sobre la imagen sin exceder su longitud, es decir, restringir la amplitud de movimiento del punto. En cuanto a la dirección de dicho movimiento, se trazó una recta perpendicular $a_{1}$ al eje $x$, utilizando la barra de entrada del programa donde se introdujo la expresión $x=5$. Una vez conocida la dirección, restaba limitar el desplazamiento que debía tener el punto sobre $a_{1}$, de manera que éste no excediera la longitud de la imagen. La representación de este conjunto de valores se hizo a través de la construcción de una circunferencia cuyo radio podía variar, lo cual condujo a la creación de un deslizador llamado a con un valor mínimo de 0.01 y máximo 1.05, valores estimados considerando el tamaño de la imagen del pañuelo, el cual sería empleado como radio de la circunferencia en cuestión.

Con respecto a la localización del centro de la circunferencia a construir, se interceptó la recta $a_{1}$ con el eje $x$ obteniendo un punto $E$. Seguidamente, se trazó una circunferencia $f$ centrada en $E$ de radio $a$ (deslizador), la cual se interceptó con la recta $a_{1}$ para obtener el punto $F$, que se desplaza al animar el deslizador, es decir, se desplaza por la imagen del paño de lana sin exceder sus límites. Como se debía insertar una imagen, eran necesarios dos puntos que tuvieran el movimiento de $F$. Para ello, se trazó una recta tangente $g$ a $f$ en el punto $F$ y posteriormente se interceptó $g$ con $d_{1} \mathrm{y} e$, obteniéndose los puntos $E_{2}=(4, a)$ y $F_{2}=(6.5, a)$, los cuales en su coordenada $y$ tienen el valor del radio de la circunferencia $f$, siendo estos puntos móviles a los cuales se les asocia la imagen del peine (Ver Figura 3).

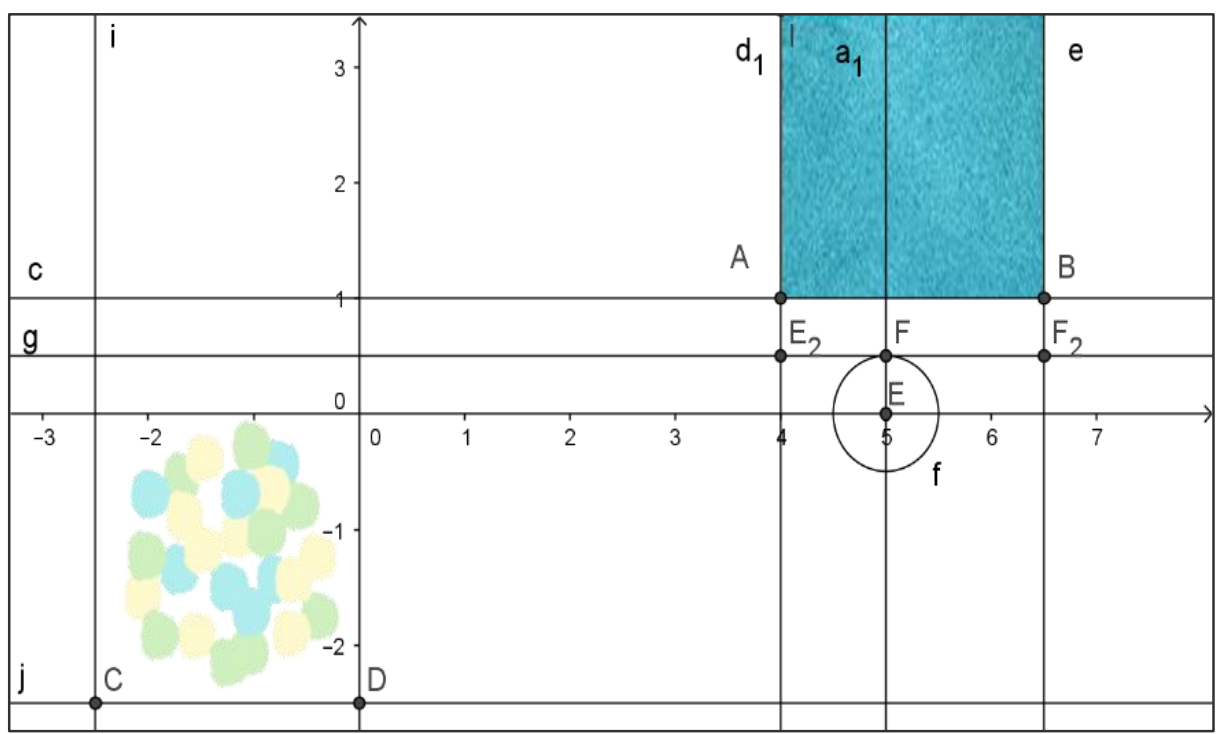

Figura - 3 Representación del movimiento para cargar el peine

Fuente: Elaborado por las autoras

\subsection{Desplazamiento del peine (Tarea No. 3)}

Esta tarea tuvo como propósito establecer la dirección por la cual transitaría la imagen del peine una vez cargado hasta la imagen del grupo de los papelillos, que permitiese visualizar 
la atracción y repulsión entre ambos objetos. Para llevar a cabo esto, se trazó un segmento $\overline{D^{\prime} F}$ que representase el recorrido de la imagen del peine. Una vez construido $\overline{D^{\prime} F}$, se aplicó una traslación a la imagen del peine, según un vector $\vec{u}$ de origen $F$ y extremo $G$, siendo este último punto creado sobre el segmento $\overline{D^{\prime} F}$, de manera que cuando $G$ se desplazara por $\overline{D^{\prime} F}$, la imagen del peine hiciese lo propio (acercarse o alejarse) a la imagen de los papelillos. Luego, con la herramienta Traslación se trasladó la imagen del peine (Ver Figura 4).

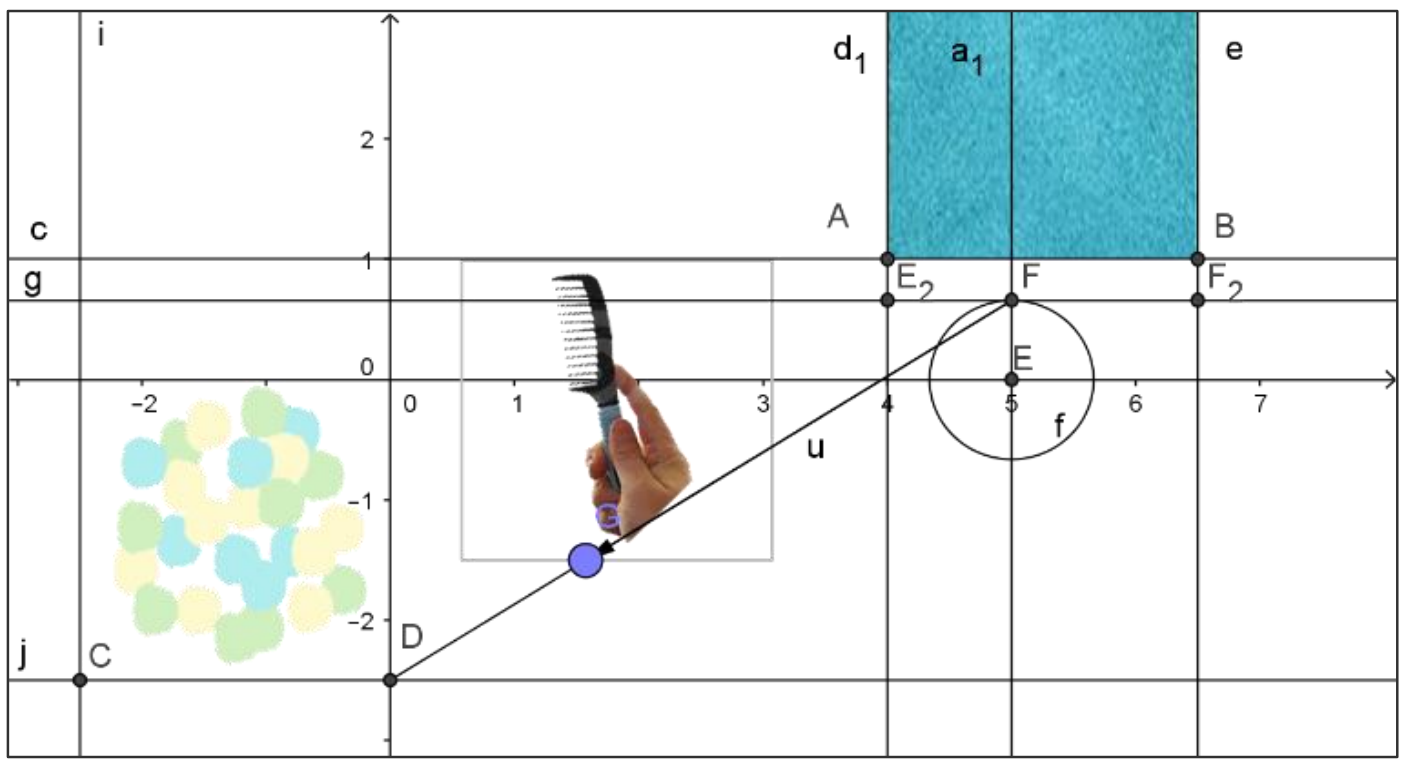

Figura - 4 Desplazamiento de la imagen del peine

Fuente: Elaborado por las autoras

\subsection{Movimiento de los papelillos (Tarea No. 4)}

El propósito de esta tarea fue representar las fuerzas de atracción y repulsión que ejerce el peine cargado al grupo de papelillos con carga neta cero. Para ello, se representaron los trozos de papel y luego el movimiento de las fuerzas mencionadas. Con respecto a los papelillos que tienen carga neta cero, primero se trazó una recta perpendicular $l$ al eje $x$, introduciendo en la barra de entrada la expresión $x=-1$, interceptando esa recta con el eje $x$, obteniendo el punto $M$, sobre el cual se construyeron tres circunferencias concéntricas $p, q$ y $r$ de radios estimados $0.35,0.75$ y 1.1 , que posteriormente se interceptaron con la recta 1 , resultando dos puntos por cada circunferencia, de los cuales se seleccionaron $N, O$ y $P$. Estos puntos representan los papelillos en su estado inicial, es decir, eléctricamente neutros (Ver Figura 5a) 


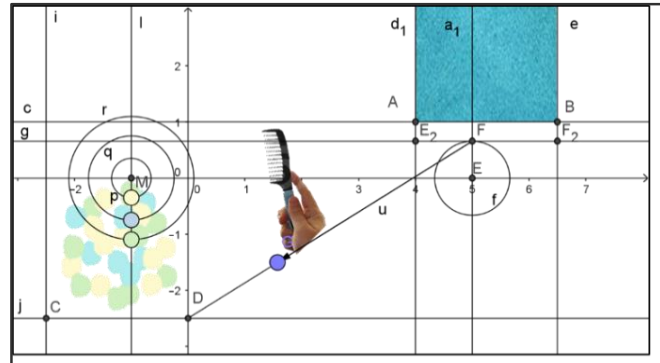

(a)

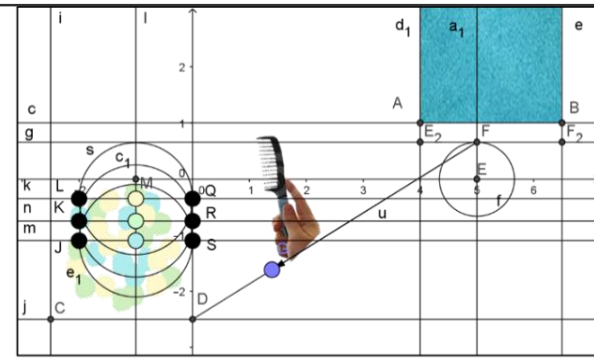

(b)

Figura - 5 Representación del movimiento de los papelillos

Fuente: Elaborado por las autoras

Para recrear el movimiento de la fuerza de atracción se necesitaba conocer tres puntos que se acercaran al peine. Para crear ese efecto, se decidió construir tres circunferencias cuyo radio variara e ubicar sobre sus bordes dos puntos de los cuales uno de ellos permitiese representar la fuerza de atracción. Esta construcción inició con la representación de tres rectas perpendiculares $n, m$ y $k$ a $l$ por los puntos $N, O$ y $P$, por las cuales se desplazarían los puntos que emularían las fuerzas. Para controlar el intervalo de valores que debían tomar los puntos, se creó un deslizador $d$ de mínimo 0 y máximo 1, valores estimados según la posición del papelillo en estado neutro y la posición final del desplazamiento del peine. Después se trazaron tres circunferencias $s, e_{1}$ y $c_{1}$ centradas en $N, O$ y $P$ y de radio $d$. Al interceptar las rectas $n$, $m$ y $e$ con las circunferencias $s, e_{1}$ y $c_{1}$ se obtuvieron los puntos $Q, R, S, J, K$ y $L$. Los tres primeros son los que recrean la fuerza de atracción del peine con los papelillos a través de la electrización por inducción y el fenómeno de polarización (Ver Figura 5b).

Para lograr la recreación del movimiento de la fuerza de repulsión se necesitaba de un tiempo en el que los electrones del peine fueran transferidos a los papelillos adheridos al peine, a través de la electrización por contacto. Para llevar a cabo esa representación, primero se precisó construir un deslizador de tipo numero llamado $T$, con mínimo 0 y máximo 2 , que representa el tiempo en segundos que demorarían los electrones en transferirse. Después se hizo necesario que iniciara el movimiento de repulsión en el momento que el deslizador $T$ llegase a su valor máximo. Para lograr esto, nos valimos de una funcionalidad dinámica del GeoGebra: los comandos (CASTILLO; PRIETO, 2018). Así, decidimos usar el comando Si $[<$ Condición $>,<$ Entonces $>$ ], que en nuestro caso su sintaxis en la barra de entrada fue la siguiente: $\operatorname{Si}[T=2, \operatorname{Valor}(d, 0)]$. Con esto, fue posible conseguir que los papelillos retornasen a su punto inicial y se pudiera recrear el movimiento de la fuerza de repulsión, en tanto que el peine y los papelillos tienen la misma carga en esa situación. 


\subsection{Posicionamiento del panel de control (Tarea No. 5)}

El valor de la distancia entre las cargas $(r)$ corresponde con la distancia entre los puntos $D$ y $G$. Para determinarla como variable se empleó un comando del programa. Para mostrar este valor en la Vista Grafica 2 se usaron textos dinámicos, opción que ofrece el GeoGebra para representar las medidas de objetos que pueden variar en tiempo real. Esta opción también fue empleada para mostrar el valor de la fuerza eléctrica, pero para conocer su valor numérico se utilizó la expresión matemática $F_{e}=K \frac{\left|q_{1}\right|\left|q_{2}\right|}{r^{2}} N$, donde $\left|q_{1}\right|\left|q_{2}\right|$ son las variables de las cargas controladas por los deslizadores $\mathrm{y}, r^{2}$ es el cuadrado de la distancia entre las cargas conocida.

Es importante destacar que el valor de estas cargas no está relacionado con la escena que se muestra en el simulador. Su valor es el que el usuario considere más conveniente comprendido entre los parámetros asociados a los deslizadores. Para controlar tales cargas se crearon cuatro deslizadores, dos asociados al número entero con mínimo de 1 y máximo 9 y los otros asociados al exponente de la carga con mínimo -9 y máximo - 1 . Los valores de las cargas debían ser números enteros, en consideración de aquella propiedad que establece que la carga eléctrica esta cuantizada. Los cuatro deslizadores fueron asociados a casillas de entradas ubicadas en la parte superior de la Vista Gráfica 2 (ver Figura 6). En esta segunda vista que dispone el GeoGebra se puede realizar cualquier construcción geométrica, sin embargo, sólo se tendrían los elementos que darían animación a la escena mostrada en el simulador.

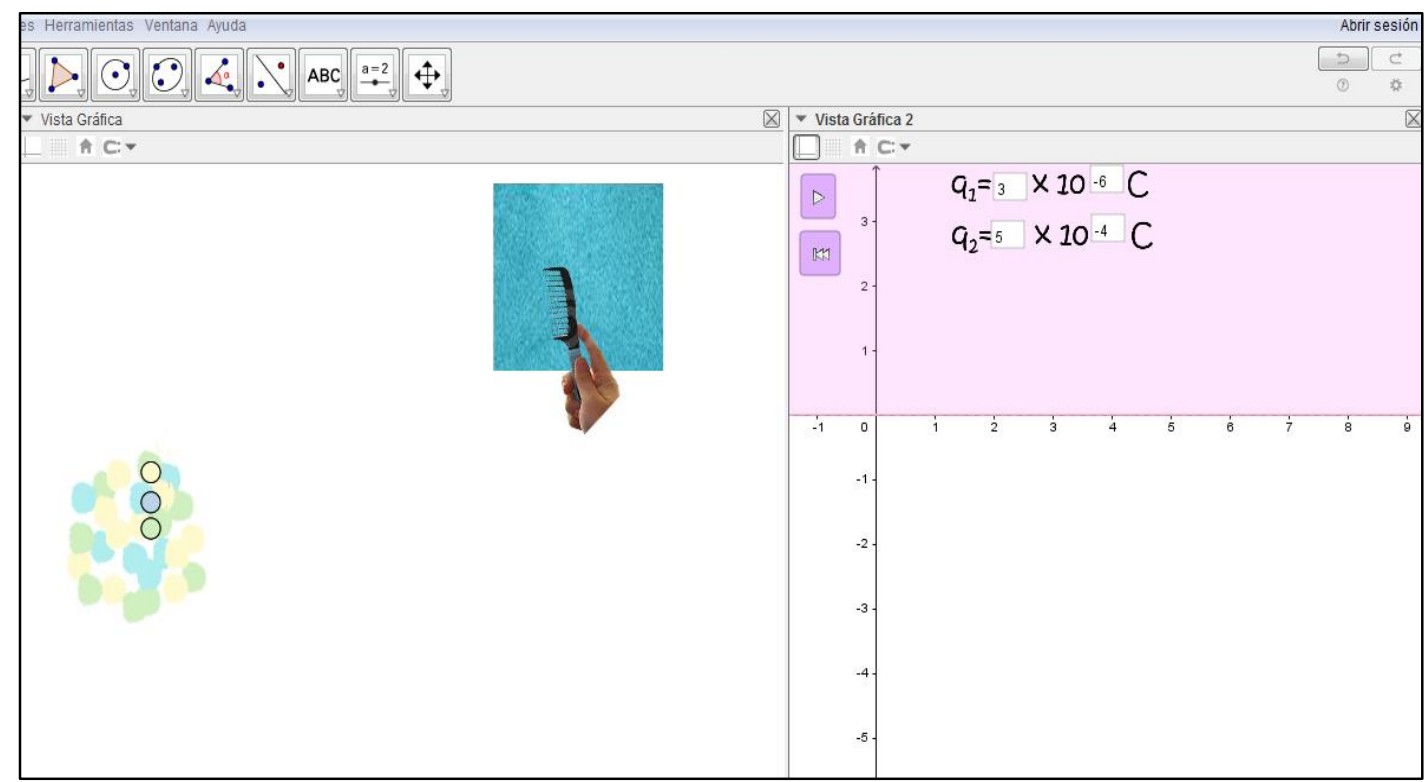

Figura - 6 Casillas de entrada en la vista grafica 2

Fuente: Elaborado por las autoras 
Seguidamente se crearon dos botones. El primero es de "inicia", el cual acciona la escena que se muestra en la parte izquierda del simulador. El segundo es de "reinicia", con el cual la escena vuelva a su estado inicial, es decir antes de cargar eléctricamente el peine. Además, se insertaron algunas imágenes para identificar cada variable. (Ver Figura 7).

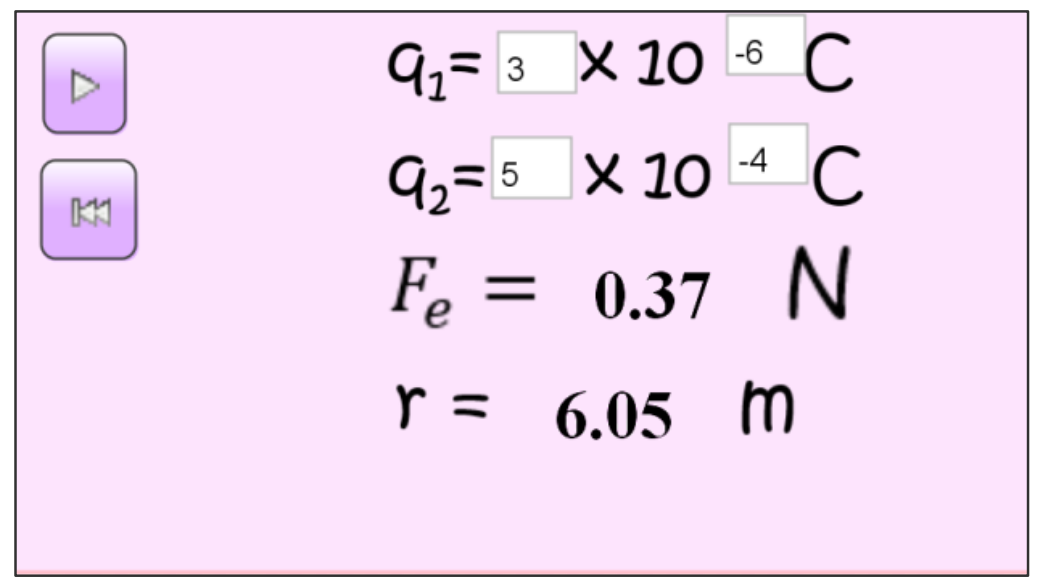

Figura 7: Panel control

Fuente: Elaborado por las autoras

\subsection{Diagrama de fuerzas de la escena (Tarea No. 6)}

El propósito de esta tarea fue mostrar el diagrama de fuerzas de la escena que se recreó en la parte derecha de la pantalla. Esto se hizo en la parte inferior de la Vista Gráfica 2 y lo primero que se hizo fue trazar una recta cualquiera $m_{1}$ en el semiplano $y<0$ y sobre ella ubicar los puntos libres $F_{1}$ y $E_{1}$, usados como centros para trazar dos círculos $r_{1}$ y $p_{1}$ de igual radio estimado 0.3 que representarían las cargas. Los círculos fueron modificados de color para distinguir los signos de las cargas: el azul para la carga positiva y el rojo para la negativa.

Estos signos serían asociados al valor de las cargas que se encuentran en la parte superior del panel de control. Luego se trazó una circunferencia $s_{1}$ centrada en $F_{1}$ y de radio 1 , producto de la intercepción entre la circunferencia $s_{1}$ con la recta $m_{1}$, obteniéndose los puntos $G_{1}$ y $H_{1}$. Después se trazaron los vectores $\overrightarrow{F_{1} H_{1}}$ y $\overrightarrow{F_{1} G_{1}}$, representando el primero la fuerza de repulsión y el segundo la fuerza de atracción. Para controlar el efecto de cambiar los vectores se utilizaron casillas de entradas, opción del software que permite ocultar y mostrar objetos construidos en el GeoGebra a conveniencia del usuario. Así, se creó una casilla para mostrar el vector que representa la fuerza de atracción y otra para la de repulsión. Estas casillas fueron asociadas con dos botones que fueron creados para controlar los colores de los círculos y para mostrar los vectores según sea el caso (Ver Figura 8). 


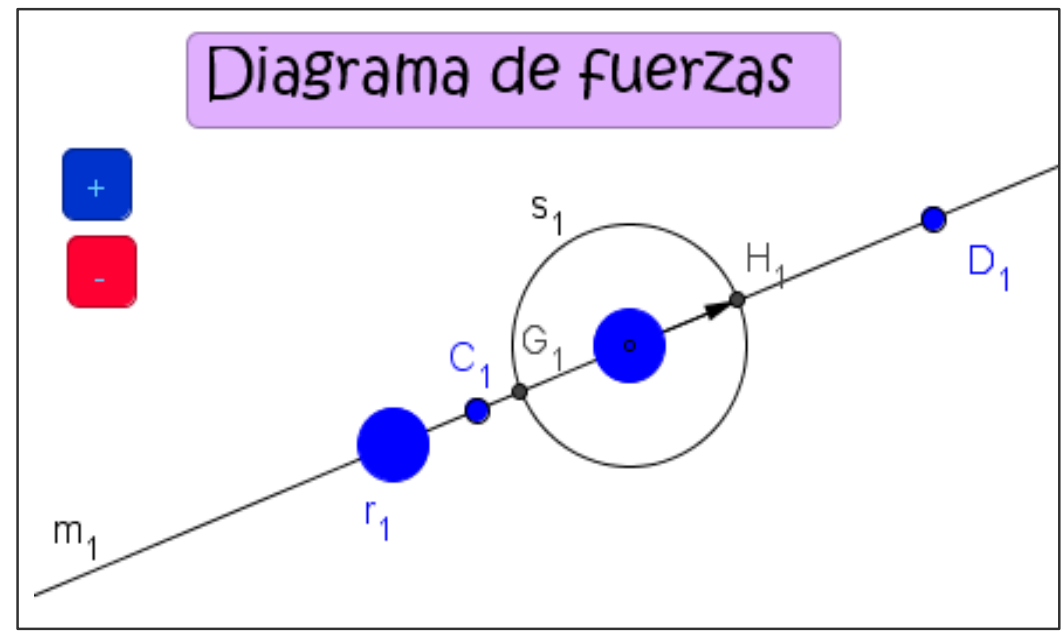

Figura - 8 Representación matemática de la escena

Fuente: Elaborado por las autoras

Finalmente se ocultaron los objetos matemáticos, siendo la apariencia final del simulador la que se muestra en la Figura 9. El simulador se encuentra disponible en https://www.geogebra.org/m/xkvhQ8wt.

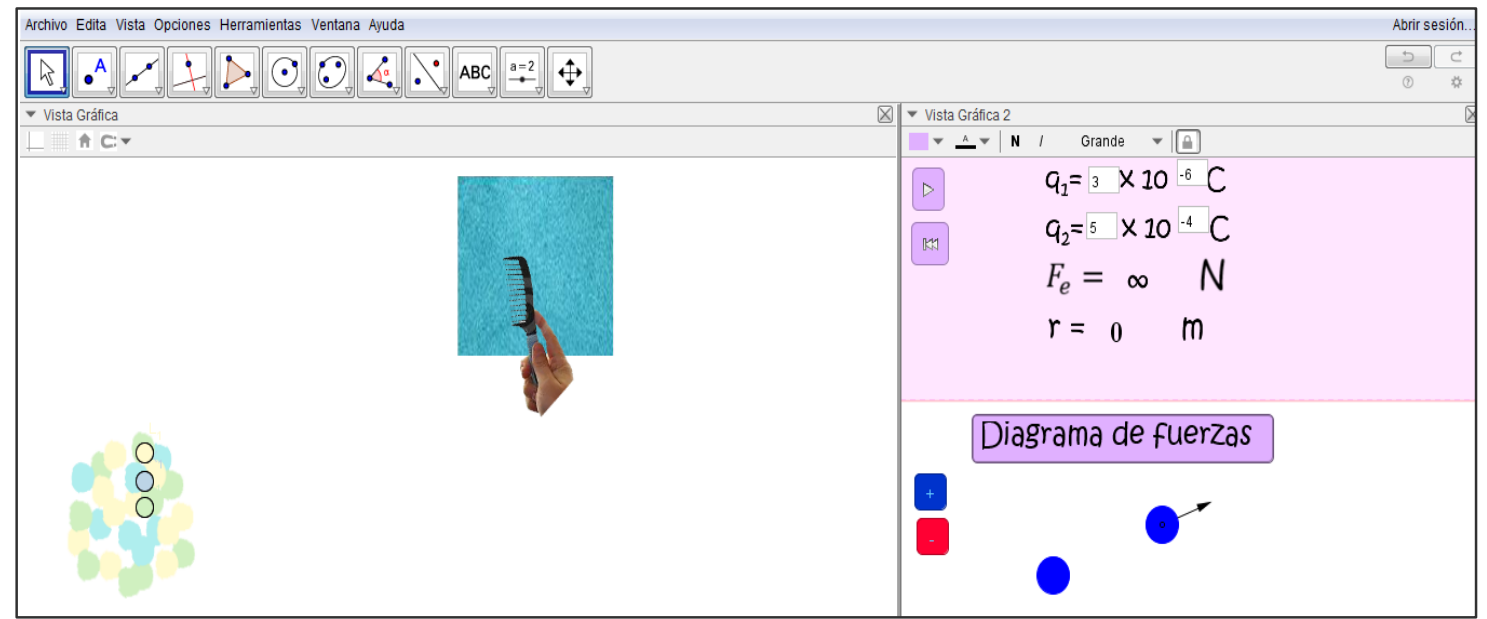

Figura - 9 Vista del usuario del simulador

Fuente: Elaborado por las autoras

\section{COMENTARIOS FINALES}

La descripción de las tareas de simulación, vinculadas a la elaboración del simulador Ley de Coulomb con GeoGebra presentadas en este trabajo, muestra los contenidos matemáticos y físicos que se precisaron en la creación de ese recurso, así como las herramientas y funcionalidades del software. En relación a las matemáticas, se resaltan los conceptos que tienen mayor presencia al momento de resolver las tareas de simulación, a saber: rectas, circunferencias, relaciones de posición de objetos geométricos en el plano, vectores y 
transformaciones en el plano. Al respecto, es importante destacar que los contenidos matemáticos no están predeterminados en la actividad, es decir, éstos surgen naturalmente en el análisis de las posibles formas de resolver la tarea de simulación. Entre esas posibles formas se opta por aquella que permita emular ese aspecto del simulador que se trabaja, de acuerdo al manejo del software.

Por su parte, los contenidos físicos que resaltan en la elaboración del simulador son: las fuerzas eléctricas que interactúan entre las cargas, los procesos de electrización, especialmente el de frotamiento, el de inducción y el efecto de polarización. Cabe destacar que, en el caso de las ciencias experimentales, como la Física, es fundamental tener conocimiento epistémico y experimental de los conceptos inmersos en la simulación, ya que las matemáticas empleadas deben responder a las características de la experimentación de la ciencia, como se mencionó anteriormente. En caso contrario, el resultado podría ser inconsistente, como señalan Rubio, Prieto y Ortiz, (2016).

En relación a la tecnología digital seleccionada, merece ser destacado el dinamismo que ofrece el GeoGebra para establecer la variación de ciertos elementos y al mismo tiempo controlar éstos, como por ejemplo, el radio de la circunferencia, lo cual favorece la visualización de los procesos de electrización en este caso. Aunado a esto, las herramientas que ofrece este software para realizar construcciones, así como algunos de sus comandos, resultan muy versátiles para elaborar este tipo de recurso, tal como es discutido por Gómez (2017).

Para finalizar, se recomienda a la comunidad de profesores de matemáticas o de ciencias naturales construir simuladores en tanto que es una experiencia que vincula el conocimiento matemático con la ciencia sustentado en tecnologías digitales. Una de las ventajas que tiene la elaboración de un recurso es que permite concebirlo de acuerdo a las características propias del grupo de estudiantes con los cuales será implementado. A su vez, son necesarias más investigaciones sobre la creación de estos recursos que permitan una mejor comprensión del conocimiento matemático puesto en juego y vinculado directamente a la ciencia que aborda la situación para emprender una simulación, así como, los alcances y limitaciones de apoyarse en ésta tecnología.

\section{REFERENCIAS}

BAEK, Y. Digital Simulation in Teaching and Learning. En D. GIBSON; Y. BAEK. (Eds.), Digital Simulations for Improving Education: Learning Through Artificial Teaching Environments (pp. 25-51), Lugar Hershey - New York. 2009 
BURBANO, V.; PINTO, J; VALDIVIESO, M. Formas de usar la simulación como un recurso didáctico. Revista Virtual Universidad Católica del Norte, n. 45, p. 16-37, 2015

CASTILlO, L. A.; PRIETO G., J. L. El Uso de Comandos y Guiones en la Elaboración de Simuladores con GeoGebra, UNIÓN, n. 52, p. 250-262, 2018

CASTILlO, L. A.; PRIETO G., J. L.; SÁNCHEZ, I. C.; GUTIÉRREZ, R. E. Uma experiência de elaboração de um simulador com GeoGebra para o ensino do movimento parabólico. PARADIGMA, v. 40, n. 2, p.196-217, 2019

CERVANTES, A.; RUBIO, L; PRIETO G., J. L. Una propuesta para el abordaje de la refracción y reflexión total interna utilizando el GeoGebra. Revista do Instituto GeoGebra de São Paulo, v. 4, n. 1, p. 18-28, 2015.

FEITOSA, M; LAVOR, O. Ensino de circuitos elétricos com auxílio de um simulador do phet. REAMEC - Rede Amazônica de Educação em Ciências e Matemática, v. 8, n. 1, p. 125-138, 2020. DOI: http://dx.doi.org/10.26571/reamec.v8i1.9014

GAMBOA, R. Uso de la tecnología en la enseñanza de las matemáticas. Cuadernos de investigación y formación en educación matemática, n. 3, p. 11-44, 2007

GÓMEZ, A. Elaboración del guion instruccional mediante la herramienta didáctica del recurso educativo digital. Via Inveniendi et Iudicandi, v. 12, n. 2, p. 149-180, 2017

GUTIÉRREZ, R. E.; PRIETO G., J. L.; ORTIZ, J. Matematización y trabajo matemático en la elaboración de simuladores con GeoGebra. Educación Matemática, v. 29, n. 2, p. 37-68 2017.

HONEY, M.; HILTON, M. Learning science through computer games and simulations. National Academies Press, 2011.

HOHENWARTER, M.; PREINER, J. Dynamic mathematics with GeoGebra. The Journal of Online Mathematics and its Applications. 2007. Acesso Disponvel em:

http://www.maa.org/external_archive/joma/Volume7/Hohenwarter/

RUBIO, L.; PRIETO G., J. L.; ORTIZ, J. La matemática en la simulación con GeoGebra. Una experiencia con el movimiento en caída libre. IJERI: International Journal of Educational Research and Innovation, v. 5, p. $90-111,2016$.

SÁNCHEZ, I.; PRIETO G., J. L. Características de las prácticas matemáticas en la elaboración de simuladores con GeoGebra. NÚMEROS: Revista de didáctica de las Matemáticas, n. 96, p. 97-101, 2017.

SERWAY, R. A. Física tomo II. México D.F.: McGraw-Hill, 1997.

UNESCO. Informe de seguimiento de la Educación Para Todos 2000-2015. Logros y Desafíos. París: UNESCO, 2015.

VILLAREAL, M. Tecnologías y educación matemática: necesidad de nuevos abordajes para la enseñanza. Revista Virtualidad, Educación y Ciencia, v.3, n. 5, p. 73-94, 2012. 
Enviado en: 29 de diciembre de 2019.

Aprobado en: 23 de marzo de 2020. 